Ю. В. Кириченко ${ }^{1}$, В. Д. Карлов ${ }^{2}$, И. Ю. Кириченко ${ }^{3}$, А. Н. Артеменко ${ }^{4}$

${ }^{1}$ Национальный научный центр “Харьковский физико-технический институт”, Харьков, Украина

${ }^{2}$ Харьковский национальный университет Воздушных Сил им. И. Кожедуба, Харьков, Украина

${ }^{3}$ Национальный технический университет Украины “КПИ им. И. Сикорского”, Киев, Украина

${ }^{4}$ Генеральный штаб Вооруженных Сил Украины, Киев, Украина

\title{
ПЛАЗМЕННАЯ АНТЕННА НА ОСНОВЕ СТРАТИФИЦИРОВАННОГО ПЛАЗМЕННОГО СТОЛБА
}

\begin{abstract}
Предмет изучения в статье является излучение плазменной антенны, в которой продольная неоднородность представляет собой страты, то есть зоны повышенной и пониженной плотности плазмы. Цель: вычисление диаграмм направленности и коэффициентов трансформации энергии поверхностной волны плазменного цилиндра в энергию излучения. Задание: анализ зависимости диаграмм направленности и коэффициентов трансформации энергии поверхностной волны от геометрических параметров антенны (радиусов плазменного цилиндра и диэлектрической оболочки), плотности плазмы, частоты излучения, диэлектрической проницаемости и др. Методы: спектральный метод, метод перевала для вычисления асимптотик интегралов, метод последовательных приближений для решения системы интегро-дифференциальных уравнений. Получены следующие результаты: показано, что при небольшом количестве страт плазменная антенна излучает под малыми углами к оси антенны. Вычислены диаграммы направленности для практически важных параметров антенны. Получены зависимости коэффициента трансформации энергии в излучение от глубины модуляции стационарных страт и страт, амплитуды которых изменяются. Выводы: проведенные в статье исследования показали, что стратифицированный плазменный столб может быть применен в качестве антенны. Максимум главного лепестка диаграммы направленности, которая имеет небольшую ширину на пол высот, и расположенный под малыми углами к оси антенны. Приведенные примеры показывают, что коэффициент превращения энергии в излучение достигает $10-30 \%$.
\end{abstract}

Ключевые слова: страты, СВЧ - излучение, холодная плазма, поверхностные волны, холодная плазма, диаграмма направленности.

\section{Введение}

Страты в газовом разряде, представляющие собой волны ионизации, образуют продольные неоднородности плотности плазменного столба $[1,2]$. Поверхностные волны в плазменном столбе, проходя через эти неоднородности, могут при определенных условиях трансформироваться в излучение. Поэтому стратифицированный столб газоразрядной плазмы может служить основой для создания одной из разновидностей плазменных антенн.

\section{Постановка задачи}

В настоящей работе исследуется преобразование аксиально-симметричной поверхностной волны в цилиндрическом столбе стратифицированной холодной изотропной плазмы в излучение. Плазма окружена однородным диэлектриком. Вблизи границ существования форма страт близка к гармонической $[1,2]$. Это обстоятельство будет использовано в настоящей работе. Метод, позволяющий решать задачи, связанные с поверхностными волнами в системах с непрерывно изменяющимися параметрами, разработан В.В. Шевченко в фундаментальных работах [3], и изложен в [4]. Согласно этому методу полное поле открытой волноведущй системы раскладывается по полному набору функций, включающему в себя поверхностные и пространственные волны. Коэффициенты такого разложения при наличии неоднородностей зависят от продольной координаты и удовлетворяют системе интегродифференциальных уравнений. Эти коэффициенты определяют амплитуды прошедшей и отраженной поверхностных волн, а также волны излучения. Отметим, что метод спектрального разложения Шевченко является математически строго обоснованным и является единственной основой теории излучения антенн поверхностных волн, свойства которых непрерывно изменяются в продольном направлении.

В статьях $[5,6]$ представлены результаты исследования планарных и цилиндрических плазменных антенн с сильной непрерывно меняющейся продольной неоднородностью плотности плазмы.

\section{Основные уравнения}

На рис. 1 показаны продольный и поперечный разрезы плазменного цилиндра с радиусом $a$, окруженного диэлектриком с диэлектрической проницаемостью $\varepsilon_{d}$ и внешним радиусом $b$. Этот плазменно-диэлектрический волновод неограничен вдоль оси $z$ ( $z$ и $\rho$ - цилиндрические координаты). На участке $0 \leq z \leq L$ находятся страты, которые моделируются с помощью гармонически изменяющейся диэлектрической проницаемости $\varepsilon_{p}(z)$. Величины $\varepsilon_{p}(z)$ и $n_{e}(z)$ связаны соотношением

$$
\varepsilon_{p}(z)=1-\omega_{p}^{2}(z) / \omega^{2},
$$

где $\omega$ - частота волны поверхностной волны, $\omega_{p}^{2}(z)=4 \pi n_{e}(z) e^{2} / m_{e}$ - плазменная частота электронной плазмы, $e, m_{e}$-заряд и масса электрона соответственно. Гармоническая зависимость $\varepsilon_{p}(z)$ от координаты на участке $0 \leq z \leq L$ в настоящей работе выбрана в виде 


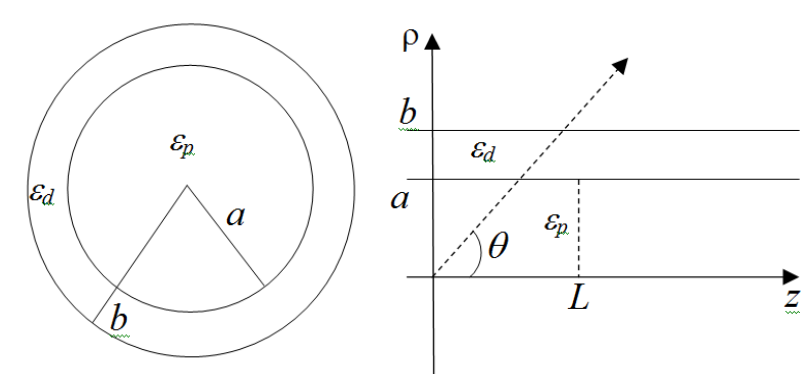

Рис. 1. Продольный и поперечный разрезы плазменно-диэлектрической структуры; радиус плазменного столба $-a$, внешний радиус диэлектрика - $b$

$$
\varepsilon_{p}(z)=-\varepsilon_{0}\left(1+\alpha \cos \left(\frac{2 \pi}{\Lambda} z+\Psi_{0}\right)\right),
$$

где $-\varepsilon_{0}<0$ - среднее по периоду значение функции $\varepsilon_{p}(z), \Lambda$-длина страты, $\Psi_{0}$ начальная фаза. Функция (1) схематично представлена на рис. 2.

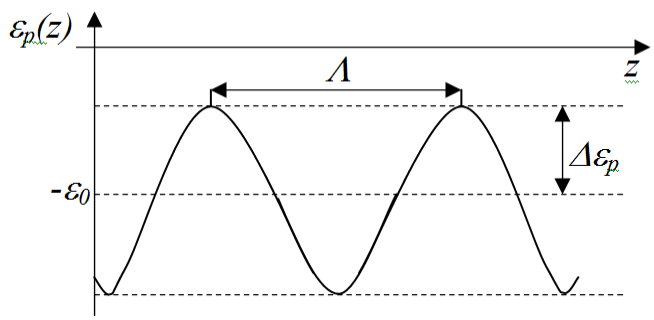

Рис. 2. Промодулированная диэлектрическая проницаемость плазмы $\varepsilon_{p}(z)$

Параметр $\varepsilon_{0}$ связан со средним по периоду значением плотности электронной плазмы $n_{e 0}$ так:

$$
\varepsilon_{0}=\frac{r_{e}}{\pi} n_{e 0} \lambda^{2}-1
$$

где $r_{e}=e^{2} / m c^{2}$ классический радиус электрона, $\lambda$ длина волны в вакууме. Очевидно, количество страт $N_{s}$ на длине неоднородности $L$ равно

$$
N_{s}=L / \Lambda
$$

Величина $\Delta \varepsilon_{p}=\varepsilon_{0}-\min \left|\varepsilon_{p}\right|$ определяет глубину модуляции $\alpha$ диэлектрической проницаемости плазмы

$$
\alpha=\Delta \varepsilon_{p} / \varepsilon_{0} .
$$

Известно [1,7], что скорость страт лежит в пределах от $10^{3}$ до $10^{6} \mathrm{~cm} / \mathrm{cек,} \mathrm{что} \mathrm{на} \mathrm{много} \mathrm{порядков}$ меньше скорости поверхностных волн в открытых плазменно-диэлектрических структурах. Кроме того, имеются эксперименты по возбуждению стоячих страт (например, [8]). Все это позволяет в нашем рассмотрении считать страты неподвижными.

В соответствии с методом спектрального разложения рассмотрим плазменный цилиндр с однородной плотностью $n_{\mathrm{e} 0}$ и, соответственно, с однородной диэлектрической проницаемостью $\varepsilon_{p}=-\varepsilon_{0}$. Временная зависимость компонент поля определя- ется множителем $\exp (-i \omega t)$. В такой плазменнодиэлектрической структуре при определенных условиях могут существовать аксиально-симметричные медленные поверхностные $E$-волны, с компонентами $\quad E_{z 0}(\rho, z), E_{\rho 0}(\rho, z), H_{\phi 0}(\rho, z) . \quad H_{\phi 0}(\rho, z)$ следующим образом зависит от координат:

$$
H_{\phi 0}(\rho, z)=\Psi_{0}(\rho) \exp \left( \pm i k_{z}^{0} z\right)
$$

где $k_{z}^{0}$ - волновой вектор поверхностной волны, $\Psi_{0}(y)$ - так называемая функция поперечного сечения поверхностной волны. Знаки + и - в (5) соответствуют распространению волны вдоль оси $z$ и против нее. Компоненты $E_{z 0}(\rho, z), E_{\rho 0}(\rho, z)$ выражаются через $H_{\phi 0}(\rho, z)$. Функция $\Psi_{0}(\rho)$ из (5) должна удовлетворять граничным условиям на границах плазменного цилиндрического столба с диэлектриком и диэлектрика с вакуумом и достаточно быстро убывать при $\rho \rightarrow \infty$. Сначала рассмотрим случай, когда волновой вектор поверхностной волны удовлетворяет условию $k<k_{z}^{0}<k \sqrt{\varepsilon_{d}}$. Из уравнений Максвелла в цилиндрических координатах и граничных условий получаем формулы для $\Psi_{0}(\rho)$ :

$$
\Psi_{0}(\rho)=\frac{1}{N_{0}}\left\{\begin{array}{lc}
K_{1}\left(\kappa_{0}^{0} \rho\right), & b \leq \rho<\infty, \\
\Delta_{1 b}^{0} J_{1}\left(\kappa_{d}^{0} \rho\right)+\Delta_{2 b}^{0} N_{1}\left(\kappa_{d}^{0} \rho\right), a \leq \rho \leq b, \\
\Delta_{1 b}^{0} / \Delta_{1 a}^{0} \cdot I_{1}\left(\kappa^{0} \rho\right), & 0 \leq \rho \leq a,
\end{array}\right.
$$

где $\left(\kappa_{0}^{0}\right)^{2}=\left(k_{z}^{0}\right)^{2}-k^{2}>0, k=\omega / c=2 \pi / \lambda$,

$$
\left(\kappa^{0}\right)^{2}=\omega_{p}^{2} / c^{2}+\left(\kappa_{0}^{0}\right)^{2}>0,\left(\kappa_{d}^{0}\right)^{2}=k^{2} \varepsilon_{d}-\left(k_{z}^{0}\right)^{2},
$$

$K_{1}(x), I_{1}(x)$ - функция Макдональда и функция Бесселя мнимого аргумента соответственно, $N_{0}-$ нормировочный множитель, который находится из условия нормировки

$$
\begin{aligned}
& \int_{0}^{\infty} d \rho \rho \frac{1}{\varepsilon(\rho)}\left(\Psi_{0}(\rho)\right)^{2}=1, \\
& \varepsilon(\rho)= \begin{cases}\varepsilon_{d}, & a \leq \rho \leq b \\
\varepsilon_{p}, & 0 \leq \rho \leq a .\end{cases}
\end{aligned}
$$

где

Формула для $N_{0}$ в (6) для краткости изложения не приводится. Коэффициенты, входящие в (6), определяются следующими соотношениями:

$$
\begin{aligned}
& \Delta_{1 a}^{0}=-\frac{\pi \kappa_{d}^{0} a}{2}\left(\frac{\kappa^{0} \varepsilon_{d}}{\kappa_{d}^{0} \varepsilon_{p}} I_{0}\left(\kappa^{0} a\right) N_{1}\left(\kappa_{d}^{0} a\right)-I_{1}\left(\kappa^{0} a\right) N_{0}\left(\kappa_{d}^{0} a\right)\right), \\
& \Delta_{1 b}^{0}=\frac{\pi \kappa_{d}^{0} b}{2}\left(\frac{\kappa_{0}^{0} \varepsilon_{d}}{\kappa_{d}^{0}} K_{0}\left(\kappa_{0}^{0} b\right) N_{1}\left(\kappa_{d}^{0} b\right)+K_{1}\left(\kappa_{0}^{0} b\right) N_{0}\left(\kappa_{d}^{0} b\right)\right), \\
& \Delta_{2 b}^{0}=-\frac{\pi \kappa_{d}^{0} b}{2}\left(\frac{\kappa_{0}^{0} \varepsilon_{d}}{\kappa_{d}^{0}} K_{0}\left(\kappa_{0}^{0} b\right) J_{1}\left(\kappa_{d}^{0} b\right)+K_{1}\left(\kappa_{0}^{0} b\right) J_{0}\left(\kappa_{d}^{0} b\right)\right) .
\end{aligned}
$$


Используя граничные условия для функции (6), получим дисперсионное уравнение для поверхностной $E$-волны:

$$
\begin{gathered}
\frac{\kappa^{0} \varepsilon_{d} I_{0}\left(\kappa^{0} a\right) N_{1}\left(\kappa_{d}^{0} a\right)-\kappa_{d}^{0} \varepsilon_{p} I_{1}\left(\kappa^{0} a\right) N_{0}\left(\kappa_{d}^{0} a\right)}{\kappa^{0} \varepsilon_{d} I_{0}\left(\kappa^{0} a\right) J_{1}\left(\kappa_{d}^{0} a\right)-\kappa_{d}^{0} \varepsilon_{p} I_{1}\left(\kappa^{0} a\right) J_{0}\left(\kappa_{d}^{0} a\right)}= \\
=\frac{\kappa_{0}^{0} \varepsilon_{d} K_{0}\left(\kappa_{0}^{0} b\right) N_{1}\left(\kappa_{d}^{0} b\right)+\kappa_{d}^{0} K_{1}\left(\kappa_{0}^{0} b\right) N_{0}\left(\kappa_{d}^{0} b\right)}{\kappa_{0}^{0} \varepsilon_{d} K_{0}\left(\kappa_{0}^{0} b\right) J_{1}\left(\kappa_{d}^{0} b\right)+\kappa_{d}^{0} K_{1}\left(\kappa_{0}^{0} b\right) J_{0}\left(\kappa_{d}^{0} b\right)}
\end{gathered}
$$

где $J_{0,1}(x), N_{.0,1}(x)$ - функции Бесселя и Неймана. Уравнение (8) содержит параметры $\varepsilon_{p}, \varepsilon_{d}, a / \lambda$ (электрический радиус плазмы), $b / a$ (относительная толщина диэлектрика) и имеет решения не при всех значениях этих величин. В некоторых же случаях уравнение (8) имеет несколько решений относительно $k_{z}^{0}$. Каждое из них соответствует определенной поверхностной волне. Ограничимся случаем, когда имеется только одна поверхностная волна.

При условии $k \sqrt{\varepsilon_{d}}<k_{z}^{0}$ выражения для компоненты поля поверхностной волны $H_{\phi 0}(\rho, z)$ отличаются от (6). В этом случае также можно получить дисперсионное уравнение. Однако оно не имеет решений, т.е. отсутствуют поверхностные волны.

Кроме поверхностной волны (5), (6) имеются также аксиально-симметричные решения уравнений Максвелла, соответствующие пространственным или псевдоповерхностным волнам [3,4]. Пространственные волны являются быстрыми. Они существуют при условии $\kappa_{0}^{2}=k^{2}-k_{z}^{2}>0$, где $k_{z}$ волновое число, $\kappa_{0}$ - поперечное волновое число. Пространственные $E$ - волны имеют компоненты $E_{z}(\rho, z), E_{\rho}(\rho, z), H_{\phi}(\rho, z)$. Азимутальная компонента магнитного поля волны

$$
H_{\phi}\left(\rho, z, \kappa_{o}\right)=\Psi\left(\rho, \kappa_{0}\right) \exp \left( \pm i k_{z} z\right) .
$$

В формуле (9) выделена зависимость от поперечного волнового числа $\kappa_{0}$. Функция поперечного сечения $\Psi\left(\rho, \kappa_{0}\right)$ пространственной $E$-волны определяется выражениями

$$
\begin{aligned}
& \Psi\left(\rho, \kappa_{0}\right)=\frac{1}{N\left(\kappa_{0}\right)} \begin{cases}u_{5} J_{1}\left(\kappa_{0} \rho\right)+u_{6} N_{1}\left(\kappa_{0} \rho\right), & b \leq \rho<\infty, \\
u_{1} J_{1}\left(\kappa_{d} \rho\right)+u_{2} N_{1}\left(\kappa_{d} \rho\right), & a \leq \rho \leq b, \\
I_{1}(\kappa \rho), & 0 \leq \rho \leq a,\end{cases} \\
& \text { где } \quad u_{6}=\frac{\pi \kappa_{0} b}{2}\left\{\frac{\kappa_{d}}{\kappa_{0} \varepsilon_{d}} u_{3} J_{1}\left(\kappa_{0} b\right)-u_{4} J_{0}\left(\kappa_{0} b\right)\right\} \\
& u_{1}=-\frac{\pi \kappa_{d} a}{2}\left\{\frac{\kappa \varepsilon_{d}}{\kappa_{d} \varepsilon_{p}} I_{0}(\kappa a) N_{1}\left(\kappa_{d} a\right)-I_{1}(\kappa a) N_{0}\left(\kappa_{d} a\right)\right\} \text {, } \\
& u_{2}=\frac{\pi \kappa_{d} a}{2}\left\{\frac{\kappa \varepsilon_{d}}{\kappa_{d} \varepsilon_{p}} I_{0}(\kappa a) J_{1}\left(\kappa_{d} a\right)-I_{1}(\kappa a) J_{0}\left(\kappa_{d} a\right)\right\},
\end{aligned}
$$

$$
\begin{gathered}
u_{3}=u_{1} J_{0}\left(\kappa_{d} b\right)+u_{2} N_{0}\left(\kappa_{d} b\right), \\
u_{4}=u_{1} J_{1}\left(\kappa_{d} b\right)+u_{2} N_{1}\left(\kappa_{d} b\right), \\
u_{5}=-\frac{\pi \kappa_{0} b}{2}\left\{\frac{\kappa_{d}}{\kappa_{0} \varepsilon_{d}} u_{3} N_{1}\left(\kappa_{0} b\right)-u_{4} N_{0}\left(\kappa_{0} b\right)\right\},
\end{gathered}
$$

$\kappa^{2}=\omega_{p}^{2} / c^{2}-\kappa_{0}^{2}, \quad \kappa_{d}^{2}=k^{2} \varepsilon_{d}-k_{z}^{2}$ (нормировочный множитель $N\left(\kappa_{0}\right)$ для краткости не приводится).

Функция (10) удовлетворяет указанным выше граничным условиям. Однако она медленно убывает при $\rho \rightarrow \infty$ и поэтому следующим образом нормирована на $\delta$ - функцию:

$$
\int_{0}^{\infty} d \rho \rho \frac{1}{\varepsilon(\rho)} \Psi\left(\rho, \kappa_{0}\right) \Psi\left(\rho, \tilde{\kappa}_{0}\right)=\delta\left(\kappa_{0}-\tilde{\kappa}_{0}\right) .
$$

Функции поперечного сечения $\Psi_{0}(y)$ и $\Psi\left(\rho, \kappa_{0}\right)$ ортогональны друг другу

$$
\int_{0}^{\infty} d \rho \rho \frac{1}{\varepsilon(\rho)} \Psi_{0}(\rho) \Psi\left(\rho, \kappa_{0}\right)=0 .
$$

Представим компоненту полного поля $H_{\phi}(\rho, z)$ в однородном слое плазмы и диэлектрике в виде спектрального разложения по полному набору функций $H_{\phi 0}(\rho, z)(5)$ и $H_{\phi}\left(\rho, z, \kappa_{0}\right)(9)$

$$
\begin{gathered}
H_{\phi}(\rho, z)=\left(B_{+}^{0} \exp \left(i k_{z}^{0} z\right)+B_{-}^{0} \exp \left(-i k_{z}^{0} z\right)\right) \Psi_{0}(\rho)+ \\
+\int_{0}^{\infty} d \kappa_{0}\left(B_{+}\left(\kappa_{0}\right) \exp \left(i k_{z} z\right)+B_{-}\left(\kappa_{0}\right) \exp \left(-i k_{z} z\right)\right) \Psi\left(\rho, \kappa_{0}\right),
\end{gathered}
$$

где первое слагаемое соответствует суперпозиции поверхностных, а второе - суперпозиции пространственных волн.

Пусть теперь на участке $0 \leq z \leq L$, как показано на рис. 1, 2, диэлектрическая проницаемость плазмы $\varepsilon_{p}$ неоднородна в продольном направлении. При этом в соответствии с методом спектрального разложения $[3,4]$ коэффициенты $B_{ \pm}^{0}, B_{ \pm}$в разложении (13) следует считать функциями координаты $z$. От $z$ теперь зависит постоянная распространения $k_{z}^{0}(z)$, являющаяся решением дисперсионного уравнения, содержащего неоднородную плотность $n_{e}(z)$. Для дальнейшего удобно сделать замену:

$$
\begin{gathered}
B_{ \pm}^{0}(z)=\frac{\exp \left( \pm i\left(\gamma_{0}(z)-k_{z}^{0} z\right)\right)}{\sqrt{k_{z}^{0}}} D_{ \pm}^{0}(z), \\
B_{ \pm}\left(\kappa_{0}, z\right)=\frac{1}{k_{z}} D_{ \pm}\left(\kappa_{0}, z\right),
\end{gathered}
$$

где $\gamma_{0}(z)=\int_{0}^{z} d z k_{z}^{0}(z)$, а $D_{ \pm}^{0}(z), D_{ \pm}\left(\kappa_{0}, z\right)-$ новые 
амплитуды поверхностных и пространственных волн. Подставляя выражение (13) с заменой (14)(15) в уравнения Максвелла и используя условия нормировки и ортогональности (7),(11),(12), получаем систему четырех интегро-дифференциальных уравнений для амплитуд $D_{ \pm}^{0}(z), D_{ \pm}\left(\kappa_{0}, z\right)$. Используя очевидные граничные условия:

$$
\begin{array}{ll}
D_{+}^{0}(z=0)=1, & D_{-}^{0}(z=L)=0, \\
D_{+}(z=0)=0, & D_{-}(z=L)=0,
\end{array}
$$

эти уравнения могут быть преобразованы в следующую систему интегральных уравнений:

$$
\begin{gathered}
D_{+}^{0}(z)=1+\int_{0}^{z} d z\left\{A_{1} D_{+}^{0}(z)+A_{2} D_{-}^{0}(z)\right\}+ \\
+\int_{0}^{z} d z \int_{0}^{\infty} d \tilde{\kappa}_{0}\left\{A_{3} D_{+}\left(\tilde{\kappa}_{0}, z\right)+A_{4} D_{-}\left(\tilde{\kappa}_{0}, z\right)\right\} \\
D_{-}^{0}(z)=\int_{L}^{z} d z\left\{\left(A_{2}\right)^{*} D_{+}^{0}(z)+A_{1} D_{-}^{0}(z)\right\}+ \\
+\int_{L}^{z} d z \int_{0}^{\infty} d \tilde{\kappa}_{0}\left\{\left(A_{4}\right)^{*} D_{+}\left(\tilde{\kappa}_{0}, z\right)+\left(A_{3}\right)^{*} D_{-}\left(\tilde{\kappa}_{0}, z\right)\right\} \\
D_{+}\left(\kappa_{0}, z\right)=\int_{0}^{z} d z\left\{A_{5} D_{+}^{0}(z)+A_{6} D_{-}^{0}(z)\right\}+ \\
+\int_{0}^{z} d z \int_{0}^{\infty} d \tilde{\kappa}_{0}\left\{A_{7} D_{+}\left(\tilde{\kappa}_{0}, z\right)+A_{8} D_{-}\left(\tilde{\kappa}_{0}, z\right)\right\} \\
D_{-}\left(\kappa_{0}, z\right)=\int_{L}^{z} d z\left\{\left(A_{6}\right)^{*} D_{+}^{0}(z)+\left(A_{5}\right)^{*} D_{-}^{0}(z)\right\}+ \\
+\int_{L}^{z} d z \int_{0}^{\infty} d \tilde{\kappa}_{0}\left\{\left(A_{8}\right)^{*} D_{+}\left(\tilde{\kappa}_{0}, z\right)+\left(A_{7}\right)^{*} D_{-}\left(\tilde{\kappa}_{0}, z\right)\right\}
\end{gathered}
$$

Величины $A_{i}$, входящие в уравнения (16)-(19) имеют смысл комплексных коэффициентов связи прошедшей поверхностной, отраженной поверхностной волн и волн излучения между собой. Эта связь возникает благодаря продольной неоднородности плазмы. Уравнения (16)-(19) и формулы для коэффициентов $A_{i}$ имеют универсальный характер и не отличаются для плоской и цилиндрической геометрии. Их вид не изменяется и при наличии диэлектрической оболочки, окружающий плазменный столб. Уравнения (16)-(19) приведены здесь для полноты изложения. Они, и формулы для $A_{i}$ приведены в $[5,6]$. Но функции $J_{0}(z), J_{1}\left(\kappa_{0}, z\right), J_{2}\left(\kappa_{0}, z\right), J_{3}\left(\tilde{\kappa}_{0}, \kappa_{0}, z\right)$, которые входят в коэффициенты $A_{i}$ будут иными. Теперь они определяются интегралами

$$
\begin{aligned}
J_{0}(z) & =\int_{0}^{\infty} d \rho \rho \frac{1}{\varepsilon_{p}(\rho)} \Psi_{0}(\rho, z)\left(\Psi_{0}(\rho, z)\right)_{z}^{\prime}, \\
J_{1}\left(\kappa_{0}, z\right) & =\int_{0}^{\infty} d \rho \rho \frac{1}{\varepsilon_{p}(\rho)} \Psi_{0}(\rho, z)\left(\Psi\left(\rho, \kappa_{0}, z\right)\right)_{z}^{\prime},
\end{aligned}
$$

$$
\begin{aligned}
& J_{2}\left(\kappa_{0}, z\right)=\int_{0}^{\infty} d \rho \rho \frac{1}{\varepsilon_{p}(\rho)} \Psi\left(\rho, \kappa_{0}, z\right)\left(\Psi_{0}(\rho, z)\right)_{z}^{\prime}, \\
& J_{3}\left(\tilde{\kappa}_{0}, \kappa_{0}, z\right)=\int_{0}^{\infty} d \rho \rho \frac{1}{\varepsilon_{p}(\rho)} \Psi\left(\rho, \kappa_{0}, z\right)\left(\Psi\left(\rho, \tilde{\kappa}_{0}, z\right)\right)_{z}^{\prime}
\end{aligned}
$$

Зависимость этих интегралов от координаты $z$ возникает из-за зависимости от $z$ корня дисперсионного уравнения $k_{z}^{0}$ и плазменной частоты $\omega_{p}$, которые содержатся в функциях $(6,10)$ (окончательные выражения для $J_{0}, J_{1}, J_{2}, J_{3}$ громоздки и для краткости не приводятся). Разложим комплексные амплитуды $D_{ \pm}^{0}(z), D_{ \pm}\left(\kappa_{0}, z\right)$ на действительные и мнимые части:

$$
\begin{aligned}
D_{+}^{0}(z)= & f_{1}(z)+i g_{1}(z), \quad D_{-}^{0}(z)=f_{2}(z)+i g_{2}(z), \\
& D_{+}\left(\kappa_{0}, z\right)=f_{3}\left(\kappa_{0}, z\right)+i g_{3}\left(\kappa_{0} z\right), \\
& D_{-}\left(\kappa_{0}, z\right)=f_{4}\left(\kappa_{0}, z\right)+i g_{4}\left(\kappa_{0} z\right) .
\end{aligned}
$$

Асимптотика интеграла в формуле (13) описывает излучение в дальней зоне. Для нахождения этой асимптотики методом перевала при $k r \rightarrow \infty$, где $r$ расстояние от участка неоднородности плазменного слоя до точки наблюдения, поступим следующим образом. Следуя работам [4, 5], перейдем к комплексной переменной $\vartheta$ с помощью формул $\kappa_{0}=k \sin \vartheta, k_{z}=k \cos \vartheta$. Контур интегрирования $C$ по комплексной переменной $\vartheta$ состоит из двух частей $C_{1}$ и $C_{2}$ как показано на рис. $3[4,5]$. Отметим, что точке $\kappa_{0}=k$ соответствует точка $\operatorname{Im}(\vartheta)=0, \quad \operatorname{Re}(\vartheta)=\pi / 2$ на этом рисунке.

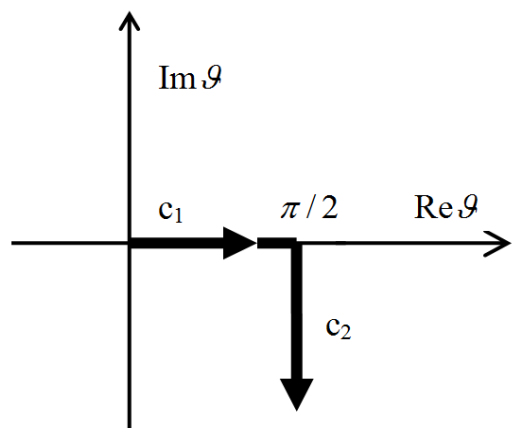

Pис. 3. Контур интегрирования $C=C_{I}+C_{2}$ по комплексной переменной $\theta$

На контуре $C$ имеются две точки перевала. Это $\vartheta=\theta$ и $\vartheta=\pi-\theta$, где $\theta$ - угол наклона радиуса вектора точки наблюдения к оси $z$ в плоскости $(\rho, z)$ (рис. 1). Эти две точки расположены на контуре $C_{1}$, который соответствует следующему интервалу изменения переменной $\kappa_{0}: 0 \leq \kappa_{0} \leq k$. Точка перевала $\vartheta=\theta$ определяет излучение под острыми углами $\theta$, а точка $\vartheta=\pi-\theta$ под тупыми углами $\theta$. Поскольку обе точки расположены на контуре $C_{1}$, мы ограничимся только этим отрезком контура $C$ при решении 
системы интегральных уравнений (16)-(19), т.е. предполагаем, что в (16)-(19) справедливо приближение

$$
\int_{0}^{\infty} d \tilde{\kappa}_{0}\{\} \approx \int_{0}^{k} d \tilde{\kappa}_{0}\{\}=k \int_{0}^{\pi / 2} d \tilde{\vartheta} \cos \tilde{\vartheta}\{\} .
$$

Предположение (27) позволяет следующим образом разложить коэффициенты $A_{i}$ на действительные и мнимые части

$$
\begin{gathered}
A_{1}(z)=a_{1}(z), A_{2}(z)=a_{2}(z)-i b_{2}(z), \\
A_{3}\left(\tilde{\kappa}_{0}, z\right)=a_{3}\left(\tilde{\kappa}_{0}, z\right)-i b_{3}\left(\tilde{\kappa}_{0}, z\right), \\
A_{4}\left(\tilde{\kappa}_{0}, z\right)=a_{4}\left(\tilde{\kappa}_{0}, z\right)-i b_{4}\left(\tilde{\kappa}_{0}, z\right), \\
A_{5}\left(\kappa_{0}, z\right)=a_{5}\left(\kappa_{0}, z\right)+i b_{5}\left(\kappa_{0}, z\right), \\
A_{6}\left(\kappa_{0}, z\right)=a_{6}\left(\kappa_{0}, z\right)-i b_{6}\left(\kappa_{0}, z\right), \\
A_{7}\left(\tilde{\kappa}_{0}, \kappa_{0}, z\right)=a_{7}\left(\tilde{\kappa}_{0}, \kappa_{0}, z\right)+i b_{7}\left(\tilde{\kappa}_{0}, \kappa_{0}, z\right), \\
A_{8}\left(\tilde{\kappa}_{0}, \kappa_{0}, z\right)=a_{8}\left(\tilde{\kappa}_{0}, \kappa_{0}, z\right)-i b_{8}\left(\tilde{\kappa}_{0}, \kappa_{0}, z\right) .
\end{gathered}
$$

Формулы для коэффициентов $A_{1}(z), A_{2}(z)$, $A_{3}\left(\tilde{\kappa}_{0}, z\right), \quad A_{4}\left(\tilde{\kappa}_{0}, z\right), \quad A_{5}\left(\tilde{\kappa}_{0}, z\right), \quad A_{6}\left(\tilde{\kappa}_{0}, z\right)$, $A_{7}\left(\tilde{\kappa}_{0}, \kappa_{0}, z\right), A_{8}\left(\tilde{\kappa}_{0}, \kappa_{0}, z\right)$ приведены в работе [6]. В этих формулах выражения для $J_{0}, J_{1}, J_{2}, J_{3}$ следует заменить на ((20)-(23)). Подставляя разложения (24)-(26), (28)-(34) в (16)-(19), и используя приближение (27), получим интегральные уравнения для действительных функций $f_{i}, g_{i}(i=1,2,3,4)$ :

$$
\begin{aligned}
& f_{1}(\tilde{z})=1+\int_{0}^{\tilde{z}} d \tilde{z}\left\{\alpha_{1}(\tilde{z}) f_{1}(\tilde{z})+\alpha_{2}(\tilde{z}) f_{2}(\tilde{z})+\beta_{2}(\tilde{z}) g_{2}(\tilde{z})\right\}+ \\
& +\int_{0}^{\tilde{z}} d \tilde{z} \int_{0}^{\pi / 2} d \tilde{\vartheta}\left\{\alpha_{3}(\tilde{\vartheta}, \tilde{z}) f_{3}(\tilde{\vartheta}, \tilde{z})+\beta_{3}(\tilde{\vartheta}, \tilde{z}) g_{3}(\tilde{\vartheta}, \tilde{z})+\right. \\
& \left.+\alpha_{4}(\tilde{\vartheta}, \tilde{z}) f_{4}(\tilde{\vartheta}, \tilde{z})+\beta_{4}(\tilde{\vartheta}, \tilde{z}) g_{4}(\tilde{\vartheta}, \tilde{z})\right\} ; \\
& g_{1}(\tilde{z})=\int_{0}^{\tilde{z}} d \tilde{z}\left\{\alpha_{1}(\tilde{z}) g_{1}(\tilde{z})+\alpha_{2}(\tilde{z}) g_{2}(\tilde{z})-\beta_{2}(\tilde{z}) f_{2}(\tilde{z})\right\}+ \\
& +\int_{0}^{\tilde{z}} d \tilde{z} \int_{0}^{\pi / 2} d \tilde{\vartheta}\left\{\alpha_{3}(\tilde{\vartheta}, \tilde{z}) g_{3}(\tilde{\vartheta}, \tilde{z})-\beta_{3}(\tilde{\vartheta}, \tilde{z}) f_{3}(\tilde{\vartheta}, \tilde{z})+\right. \\
& \left.+\alpha_{4}(\tilde{\vartheta}, \tilde{z}) g_{4}(\tilde{\vartheta}, \tilde{z})-\beta_{4}(\tilde{\vartheta}, \tilde{z}) f_{4}(\tilde{\vartheta}, \tilde{z})\right\} \\
& f_{2}(\tilde{z})=\int_{1}^{\tilde{z}} d \tilde{z}\left\{\alpha_{2}(\tilde{z}) f_{1}(\tilde{z})-\beta_{2}(\tilde{z}) g_{1}(\tilde{z})+\alpha_{1}(\tilde{z}) f_{2}(\tilde{z})\right\}+ \\
& +\int_{1}^{z} d \tilde{z} \int_{0}^{\pi / 2} d \tilde{\vartheta}\left\{\alpha_{4}(\tilde{\vartheta}, \tilde{z}) f_{3}(\tilde{\vartheta}, \tilde{z})-\beta_{4}(\tilde{\vartheta}, \tilde{z}) g_{3}(\tilde{\vartheta}, \tilde{z})+\right. \\
& \left.+\alpha_{3}(\tilde{\vartheta}, \tilde{z}) f_{4}(\tilde{\vartheta}, \tilde{z})-\beta_{3}(\tilde{\vartheta}, \tilde{z}) g_{4}(\tilde{\vartheta}, \tilde{z})\right\} \\
& g_{2}(\tilde{z})=\int_{1}^{\tilde{z}} d \tilde{z}\left\{\alpha_{2}(\tilde{z}) g_{1}(\tilde{z})+\beta_{2}(\tilde{z}) f_{1}(\tilde{z})+\alpha_{1}(\tilde{z}) g_{2}(\tilde{z})\right\}+ \\
& +\int_{1}^{\bar{z}} d \tilde{z} \int_{0}^{\pi / 2} d \tilde{\vartheta}\left\{\alpha_{4}(\tilde{\vartheta}, \tilde{z}) g_{3}(\tilde{\vartheta}, \tilde{z})+\beta_{4}(\tilde{\vartheta}, \tilde{z}) f_{3}(\tilde{\vartheta}, \tilde{z})+\right. \\
& \left.+\alpha_{3}(\tilde{\vartheta}, \tilde{z}) g_{4}(\tilde{\vartheta}, \tilde{z})+\beta_{3}(\tilde{\vartheta}, \tilde{z}) f_{4}(\tilde{\vartheta}, \tilde{z})\right\}
\end{aligned}
$$


В уравнениях (35)-(42) величина $\vartheta$ связана с углом наблюдения, а $\tilde{\vartheta}$ - переменная интегрирования. Найдя асимптотику интеграла, входящего в разложение (13), и соответствующий вектор Пойнтинга, получаем угловое распределение мощности излучения под острыми $P^{+}(\theta)$ и тупыми углами $P^{-}(\theta)$ :

$$
\begin{gathered}
\frac{d P^{+}(\theta)}{d \theta}=\frac{c}{4 k}\left(f_{3}^{2}(\theta, 1)+g_{3}^{2}(\theta, 1)\right), \\
\frac{d P^{-}(\theta)}{d \theta}=\frac{c}{4 k}\left(f_{4}^{2}(\pi-\theta, 0)+g_{4}^{2}(\pi-\theta, 0)\right) .
\end{gathered}
$$

Амплитуды $f_{1}(\tilde{z}), g_{1}(\tilde{z}), f_{2}(\tilde{z}), g_{2}(\tilde{z})$ определяют поле поверхностной волны и вектор Пойнтинга, который определяет мощности $P_{0}^{+}(z)$ и $P_{0}^{-}(z)$ прошедшей и отраженной поверхностных волн соответственно. Коэффициенты прохождения $\eta_{0}^{+}$и отражения $\eta_{0}^{-}$для поверхностной волны равны:

$$
\begin{aligned}
& \eta_{0}^{+}=P_{0}^{+}(z \rightarrow \infty) / P_{0}^{+}(z \rightarrow-\infty)=\left(f_{1}^{2}(1)+g_{1}^{2}(1)\right), \\
& \eta_{0}^{-}=P_{0}^{-}(z \rightarrow \infty) / P_{0}^{+}(z \rightarrow-\infty)=\left(f_{2}^{2}(0)+g_{2}^{2}(0)\right) .
\end{aligned}
$$

Соотношения (43), (44) позволяют найти диаграммы направленности излучения, а также коэффициенты $\eta^{+}$и $\eta^{-}$преобразования энергии поверхностной волны в энергию излучения под острыми и тупыми углами $\theta$ соответственно:

$$
\begin{gathered}
\eta^{+}=\left(1 / P_{0}^{+}(z \rightarrow-\infty)\right) \times \\
\times \int_{0}^{\pi / 2} d \theta \frac{d P^{+}(\theta)}{d \theta}=\int_{0}^{\pi / 2} d \theta\left(f_{3}^{2}(\theta, 1)+g_{3}^{2}(\theta, 1)\right) \\
\eta^{-}=\left(1 / P_{0}^{+}(z \rightarrow-\infty)\right) \times \\
\times \int_{\pi}^{\pi / 2} d \theta \frac{d P^{-}(\theta)}{d \theta}=\int_{\pi}^{\pi / 2} d \theta\left(f_{4}^{2}(\pi-\theta, 0)+g_{4}^{2}(\pi-\theta, 0)\right) .
\end{gathered}
$$

\section{Результаты вычислений}

Для решения системы интегральных уравнений (35)-(42) был использован метод последовательных приближений. Нулевое приближение для амплитуд определяется следующими соотношениями:

$$
\begin{aligned}
& f_{1}(\tilde{z})=1, g_{1}(\tilde{z})=f_{2}(\tilde{z})=g_{2}(\tilde{z})=g_{2}(\tilde{z})= \\
= & f_{3}(\vartheta, \tilde{z})=g_{3}(\vartheta, \tilde{z})=f_{4}(\vartheta, \tilde{z})=g_{4}(\vartheta, \tilde{z})=0 .
\end{aligned}
$$

Формулы (47) соответствуют прохождению поверхностной волны через участок неоднородной плазмы без искажения. Условия существования единственной поверхностной волны не могут быть получены в аналитическом виде и в каждом отдельном случае находятся численно, в виде ограничений на параметры $\varepsilon_{p}, \varepsilon_{d}, a / \lambda, b / a$. Последние три параметра определяют минимальное значение модуля диэлектрической проницаемости однородной плаз- мы $\varepsilon_{p r}$, при котором еще существует поверхностная волна. Очевидно, должно выполняться условие

$$
\varepsilon_{0}>\varepsilon_{p r} .
$$

Величина $\varepsilon_{p r}$ немонотонно зависит от параметров $\varepsilon_{d}, a / \lambda, b / a$ и может быть найдена только в результате численного решения уравнения (8). Глубина модуляции (4) должна подчиняться условию

$$
\alpha \leq \alpha_{\max },
$$

где $\alpha_{\max }-$ максимально допустимое значение $\alpha$. Величина $\alpha_{\max }$ определяется соотношением $\alpha_{\max }=\left(\varepsilon_{0}-\varepsilon_{p r}\right) / \varepsilon_{0}$, которое следует из (4) и определения параметра $\varepsilon_{p r}$. Величина $\alpha_{\max }$ зависит не только от параметров $\varepsilon_{d}, a / \lambda, b / a$, но также, как видно из (2), от средней плотности плазмы $n_{e 0}$ и радиуса плазменного столба $a$. Последний определяет длину волны $\lambda$ при заданном параметре $a / \lambda$. Итак, параметры задачи должны выбираться из условий (48),(49).

В настоящей работе проведены вычисления коэффициентов преобразования энергии, среди которых наиболее важными для физики антенн являются коэффициенты $\eta^{+}$(45) и $\eta^{-}$(46), поскольку именно они определяют эффективность излучения предлагаемой модели плазменной антенны. Точность вычислений, определяемая разностной аппроксимацией интегралов в уравнениях (35)-(42) и от того, насколько справедливо приближение (27), контролировалась с помощью очевидного равенства $\eta_{0}^{+}+\eta_{0}^{-}+\eta^{+}+\eta^{-}=1$.

Вычисления выполнены для нескольких наборов параметров $n_{e 0}, L / \lambda, \varepsilon_{d}, a, a / \lambda, b / a$, а также разных значений глубины модуляции $\alpha$, количества страт $N_{s}(3)$ и фазы $\Psi_{0}$. Для иллюстрации выбраны такие параметры: набор I - $n_{e 0}=10^{12} \mathrm{~cm}^{-3}, L / \lambda=3$, $\varepsilon_{d}=2.5, a=5 \mathrm{~cm}, a / \lambda=0.15, b / a=1.1$; набор II $n_{e 0}=10^{12} \mathrm{~cm}^{-3}, \quad L / \lambda=2, \quad \varepsilon_{d}=10, \quad a=1 \mathrm{~cm}$, $a / \lambda=0.02, b / a=2$; набор III - $n_{e 0}=10^{11} \mathrm{~cm}^{-3}$, $L / \lambda=3, \quad \varepsilon_{d}=10, a=5 \mathrm{~cm}, \quad a / \lambda=0.15, b / a=2$; набор IY $-n_{e 0}=10^{11} \mathrm{~cm}^{-3}, \quad L / \lambda=2, \quad \varepsilon_{d}=2.5$, $a=5 \mathrm{~cm}, a / \lambda=0.1, b / a=1.1$. Для определенности выберем длину участка неоднородности, равную $L=100$ см $[2,7]$. Тогда длина волны излучения для наборов параметров I и III будет равна $\lambda=33,3$ см., а для наборов II и IY $-\lambda=50$ см. Для того, чтобы $\lambda$ было другим, параметры задачи должны отличаться от приведенных выше.

Общие результаты для выбранных параметров сводятся к следующему. Коэффициенты $\eta^{+}$и $\eta^{-}$немонотонно зависят от количества страт $N_{s}$ при фиксированных значениях прочих параметров задачи. Коэффициент $\eta^{+}$имеет максимум при небольших значениях 
$N_{s}=1,2$, а коэффициент $\eta^{-}$имеет максимум при значениях $N_{s}=3-5$. Поэтому для излучения под острыми углами $\theta$ (рис.1) нужно небольшое число страт $N_{s}$, а для излучения под тупыми углами $\theta$ величина $N_{s}$ должна быть больше. Обозначим эти максимальные по $N_{s}$ величины $\eta^{+}$и $\eta^{-}$, как $\max \left(\eta^{+}\right)$и $\max \left(\eta^{-}\right)$соответственно. Эти величины при прочих фиксированных параметрах растут при увеличении диэлектрической проницаемости $\varepsilon_{d}$ и уменьшаются при увеличении плотности плазмы $n_{e 0}$.

В работе [9] описаны страты с глубиной модуляции $\alpha$ (4), близкой к единице. При увеличении $\alpha$ увеличивается степень продольной неоднородности плазмы. Поэтому, как и следовало ожидать, коэффициенты преобразования энергии поверхностной волны в излучение при этом растут. В работах $[5,6]$ описана сходная ситуация, когда эффективность
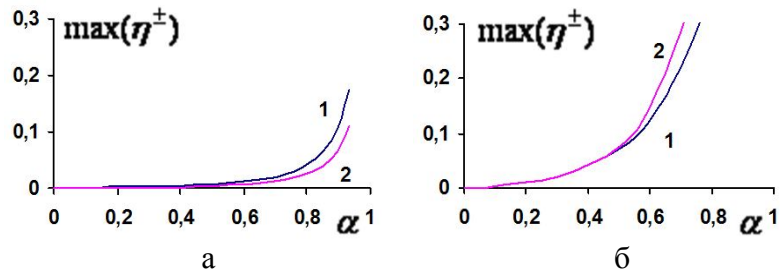

Рис. 4. Зависимость максимальных по количеству страт коэффициентов трансформации от глубины модуляции диэлектрической проницаемости $\alpha$. Кривые 1 соответствуют коэффициенту $\max \left(\eta^{+}\right)$, кривые 2 - max $\left(\eta^{-}\right)$; вычисления выполнены с наборами параметров I(a), II(б), III(в), IY(г)

Нормированные диаграммы направленности (НДН) имеют один хорошо выраженный лепесток с малым уровнем боковых лепестков при изменении количества страт в интервале $1 \leq N_{s} \leq N_{s \max }$, где $N_{s \max }=5-6$. При $N_{s} \geq N_{s \max }$ у НДН появляется множество боковых лепестков. Для иллюстрации на рис. 5 представлена НДН, вычисленная с набором параметров I при $\Psi_{0}=\pi / 2, \alpha=0.5$ и двух значений $N_{s}$.

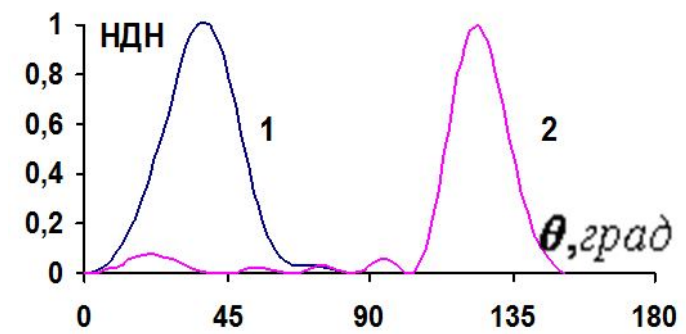

Рис. 5. НДН, вычисленные с набором параметров I при $\Psi_{0}=\pi / 2, \alpha=0.5$ и $N_{s}=1$ (кривая 1$), N_{s}=5$ (кривая 2)

Общие свойства НДН, вычисленных при этих и других значениях этих параметров, таковы. При увеличении числа страт $N_{s}$ угол $\theta_{m}$, на который приходится максимум НДН, монотонно смещается в сторону больших значений. При изменении глубины модуляции страт $\alpha$ и фазы $\Psi_{0}$, при неизменных остальных параметрах, угол $\theta_{m}$ и полуширина НДН $\Delta \theta$ изменяются незначительно. Отсюда следует, что при заданных геометрии плазменной антенны, свойствах плазмы и диэлектрика и небольшом количестве страт, можно получить излучение под острыми углами с
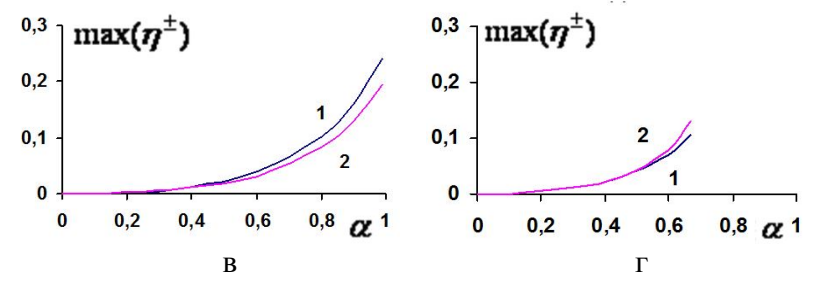

преобразования энергии поверхностной волны в излучение растет при увеличении степени неоднородности плазмы при увеличении крутизны продольного уменьшения плотности плазмы. На рис. 4 представлено поведение величин $\max \left(\eta^{+}\right)$и $\max \left(\eta^{-}\right)$как функций $\alpha$ для приведенных выше наборов параметров I, II, III, IY и для начальной фазы, равной $\Psi_{0}=\pi / 2$. Интересно отметить, что при изменении $\alpha$ количества страт $N_{s}$, на которые приходятся максимумы коэффициентов $\eta^{+}$и $\eta^{-}$остаются одними и теми же. Для штыревых антенн поверхностной волны наиболее интересно излучение под острыми углами $\theta$ (рис. 1). Видно, что коэффициенты $\max \left(\eta^{+}\right)$для выбранных нами параметров и $\Psi_{0}=\pi / 2$ достигают $10-30 \%$. Усредненные по фазе $\Psi_{0}$ значения $\max \left(\eta^{+}\right)$мало отличаются от этих величин.

малым уровнем боковых лепестков. При этом эффективность излучения, угол $\theta_{m}$ и полуширина НДН $\theta_{m}$ слабо зависят от глубины модуляции страт $\alpha$.

\section{Выводы}

Исследована трансформация энергии поверхностной волны на продольных неоднородностях цилиндрического плазменного столба, образованных стратами. Плазму окружает однородный диэлектрик. Для анализа таких открытых волноведущих систем эффективным является метод спектрального разложения Шевченко, который приводит к системе интегральных уравнений. Решение этих уравнений позволяет определить коэффициенты трансформации энергии поверхностной волны в излучение, а также вычислить диаграммы направленности. Проанализирована зависимость результатов от геометрии рассмотренной системы, свойств плазмы, диэлектрика, от количества страт и глубины модуляции плазмы. При небольшом количестве страт излучение направлено под острыми углами к оси плазменного столба. При этом уровень боковых лепестков мал. Эффективность преобразования энергии поверхностной волны в излучение составляет для выбранных нами параметров задачи $10-30 \%$. Углы, приходящиеся на максимумы диаграмм направленности, и полуширины последних слабо зависят от глубины модуляции плотности плазмы.

Таким образом, рассмотренный стратифицированный плазменный цилиндр может служить основой для создания эффективной плазменной антенны с приосевым излучением. 
1. Колебания и неустойчивости низкотемпературной плазмы / А. В. Недоспасов, В. Д. Хаит. - М.: Наука, 1979.

2. Пекарек Л. Ионизационные волны (страты) в разрядной плазме / Пекарек Л. // УФН. - 1968. - Т.94. вып. 3. - С. 463.

3. Шевченко В.В. // Акустический журнал. - 1963. - Т.9. - № 2. -С. 215.

4. Плавные переходы в открытых волноводах. Введение в теорию / В.В. Шевченко. - М.:Наука, 1969.

5. Кириченко Ю.В. // Радиотехника и электроника. - 2017. - Т.62. №12. - С. 1215.

6. Цилиндрическая плазменная антенна с сильной продольной неоднородностью / Кириченко Ю.В. // Радиотехника и электроника. - 2018. - Т.63. №5. - С. 433 - 441.

7. Зайцев А. А., Швилкин Б. Н. // Радиотехника и электроника. - 1967. - Т.12. №4. - С.736.

8. Клярфельд Б. Н .// Журнал экспериментальной и теоретической физики. - 1952. - Т.22. вып.1. - С.66.

9. Lee O.A, Bletzinger P, Garscadden A.//Journ. of Applied Physics. - 1966. - v.37. №1. - P.377.

\section{REFERENCES}

1. Nedospasov, A. V. and Khait, V. D. (1979) “"Kolebaniya i neustoychivosti nizkotemperaturnoy plazmyi” [Oscillation and instability of low temperature plasma ], Science, .

2. Pekarek, L. (1968), "Ionizatsionnyie volnyi (stratyi) v razryadnoy plazme"[Ionization waves (strata) in discharge plasma], Uspekhi Fizicheskikh Nauk, V.94, No. 3, P.463.

3. Shevchenko, V.V. (1963), Acoustic journal,V.9, No. 2, P.215.

4. Shevchenko, V.V. (1969), " Plavnyie perehodyi v otkryityih volnovodah. Vvedenie v teoriyu” [Smooth transitions in open waveguides. Introduction to theory], Science, Moscow.

5. Kirichenko, Yu.V. (2017), Radio engineering and electronics, V.62, No 12, P. 1215.

6. Kirichenko, Yu.V. (2018), “ Tsilindricheskaya plazmennaya antenna s silnoy prodolnoy neodnorodnostyu” [Cylindrical plasma antenna with a strong longitudinal inhomogeneity], Radio engineering and electronics, V.63, No5, pp. $433-441$.

7. Zaitsev, A.A. and Shvilkin, B. N. (1967), Radio engineering and electronics, V.12, No 4, P.736.

8. Klyarfeld, B.N. (1952), Journal of Experimental and Theoretical Physics, V.22, issue 1, P.66.

9. Lee, O.A, Bletzinger, P. and Garscadden, A. (1966), Journ. of Applied Physics, V.37, No1, P.377.

Рецензент: д-р техн. наук, проф. Л. Ф. Купченко, Харківський національний університет Повітряних Сил імені Івана Кожедуба, Харків Received (Надійшла) 12.05.2018 Accepted for publication (Прийнята до друку) 23.07.2018

\section{Плазмова антена на основі стратифікованого плазмового стовпа}

\section{Ю. В. Кириченко, В. Д. Карлов, І. Ю. Кириченко, А. М. Артеменко}

Предметом вивчення в статті є випромінювання плазмової антени, в якій поздовжня неоднорідність являє собою страти, тобто зони підвищеної та низької щільності плазми. Метою $є$ обчислення діаграм спрямованості та коефіцієнтів перетворення енергії поверхневої хвилі плазмового циліндра в енергію випромінювання. Завдання: аналіз залежності діаграм спрямованості та коефіцієнтів перетворення енергії поверхневої хвилі від геометричних параметрів антени (радіусів плазмового циліндра та діелектричної оболонки), щільності плазми, частоти випромінювання, діелектричної проникності та ін. Використовуваними є методи спектрального методу, метод перевалу для обчислення асимптотик інтегралів, метод послідовних наближень для розв'язання системи інтегро-диференціальних рівнянь. Отримані наступні результати. Показано, що при невеликій кількості страт плазмова антена випромінює під малими кутами до осі антени. Обчислено діаграми спрямованості для практично важливих параметрів антени. Отримано залежності коефіцієнта перетворення енергії у випромінювання від глибини модуляції стаціонарних страт та страт, амплітуди яких змінюються. Висновки. Проведені в статті дослідження показали, що стратифіцірований плазмовий стовп може бути застосованим в якості антени. Максимум головної пелюстки діаграми спрямованості, що має невелику ширину на пів висот, і розташований під малими кутами до осі антени. Наведені приклади показують, що коефіцієнт перетворення енергії у випромінювання досягає 10 - 30 \%.

Ключов і слов а: страти, НВЧ - випромінювання, холодна плазма, поверхневі хвилі, холодна плазма, діаграма спрямованості.

\section{Plasma antenna on the basis of the stratified plasma post \\ Yu. Kirichenko, V. Karlov, I. Kirichenko, A. Artemenko}

The subject of the study in the article is the radiation of a plasma antenna, in which the longitudinal heterogeneity is a stratum, that is, the zone of high and low density of the plasma. The aim is to calculate the directivity patterns and co-factors of the transformation of the surface plasma energy of the plasma cylinder into the energy of radiation. Objective: An analysis of the dependence of the directional diagrams and the coefficients of the transformation of the energy of the surface wave from the geometric parameters of the antenna (plasma cylinder radii and dielectric shell), plasma density, radiation frequency, dielectric permeability, and others. The methods used are the spectral method, the pass method for calculating the asymptotic integral, the method of successive approximations for the solution of the system of introspection equations. The following results are obtained. It is shown that with a small number of strata, the plasma antenna radiates at small angles to the antenna axis. The directional diagram for practically important antenna parameters is calculated. The dependences of the energy conversion factor in the radiation on the depth of modulation of stationary stratums and stratums, whose amplitudes change, are obtained. Conclusions. The studies carried out in the article showed that the stratified plasma column can be used as an antenna. The maximum of the main petal of the directional pattern, which has a small width of half the heights, and is located at small angles to the antenna axis. The above examples show that the energy conversion factor in radiation reaches $10-30 \%$.

Keywords: striations, microwave radiation, cold plasma, surface waves, cold plasma, directional pattern. 